\title{
Analysis of the efficiency of earthquake prediction based on the anomalous behavior of ionospheric parameters on the eve of earthquakes in the Kamchatka region
}

\author{
Vadim Bogdanov ${ }^{1, *}$ and Aleksey Pavlov ${ }^{1, * *}$ \\ ${ }^{1}$ Institute of Cosmophysical Research and Radio Wave Propagation FEB RAS, Paratunka, Russia
}

\begin{abstract}
A method is presented for short-term prediction of strong earthquakes, in which the precursors are considered the excess of current values of $f \mathrm{oF} 2$ critical frequency of the ionospheric $\mathrm{F} 2$ layer over the median values in periods of perturbed state of the magnetosphere, the appearance of ionospheric disturbances: K-layer, Es-spread F-spread, the stratification of the F2 layer, Es is the $\mathrm{r}$ type. As predicted earthquakes were considered earthquakes with magnitudes $M \geq 5.0$. Assessment of the effectiveness of the forecast was carried out in the spring and autumn periods for 2013-2017 according to the methods of A. A. Gusev and G. M. Molchan. It is shown that the method under consideration has the best prognostic efficiency for seismic events with $M \geq 6.5$ magnitude.
\end{abstract}

\section{Introduction}

The study of solar-terrestrial relations revealed a direct relationship between solar activity and processes in the magnetosphere, ionosphere and lithosphere. However, there is a feedback that determines the influence of lithospheric processes of seismic regions on the ionosphere and magnetosphere. We can say that at the ionospheric and magnetospheric altitudes there is a kind of mapping and averaging of processes occurring in the earth's crust in areas of hundreds of kilometers. Any small impact on the ionosphere can lead to trigger effects with energy release significantly exceeding the energy of primary disturbance factors of solar or lithospheric origin. Modern research has established that the earth's crust affects the physical processes occurring in the upper geospheric shells. Therefore, in seismically active regions, any anomalous changes in the behavior of ionospheric parameters formed against the background of regular daily changes in the characteristics of the ionosphere due to the influence of the Sun can provide information about the processes of earthquake preparation [1-3]. In turn, each seismic region is characterized by its most informative features (anomalies) in the behavior of the ionospheric parameters, which can be identified with the precursors of earthquakes. In the Kamchatka region, such features as long-term studies of the ionospheric parameters dynamics on the eve of the earthquake, it can include the following [4-6]:

1. precipitation from several hours to several days before the earthquake of charged particles from radiation belts into the ionosphere (formation of a K-layer);

\footnotetext{
*e-mail: vbogd@ikir.ru

**e-mail: pavlov@ikir.ru
} 
2. formation of the diffusion sporadic layer Es (Es-spread) 1-3 days before the earthquake;

3. for 1-5 days on the background of the development of magnetic storms (in autumn and spring) abnormal increase in the critical frequency $f \mathrm{oF} 2$ (increase in electron concentration), exceeding the median values (in the normal development of the magnetospheric storm in the ionosphere due to the vortex electric field there is a drift, which leads to the displacement of electrons to great heights and a decrease in their concentration).

4. formation of a diffusion layer F2 (F2-spread) lasting several hours in 1-3 days against the background of a quiet magnetosphere;

5. for 1-3 days stratification layer F2 in frequency and height (mode "H"), the so-called moving ionospheric disturbances.

\section{Short-term earthquake prediction method based on anomalous behavior of ionospheric parameters}

The paper uses the data of radiophysical observations performed by means of vertical radiosonding. Automatic ionospheric station (AIS) of vertical radiosonde is located in Paratunka village $\left(\varphi=52.97^{\circ} \mathrm{N}, \lambda=158.25^{\circ} \mathrm{E}\right)$. Observations are conducted once every 15 minutes in pulse mode at frequencies from 1 to $15 \mathrm{MHz}$. As an example, Fig. 1 shows ionogramms containing anomalies corresponding to features 4) (Fig. 1a) and 5) (Fig. 1b), listed in the previous section.

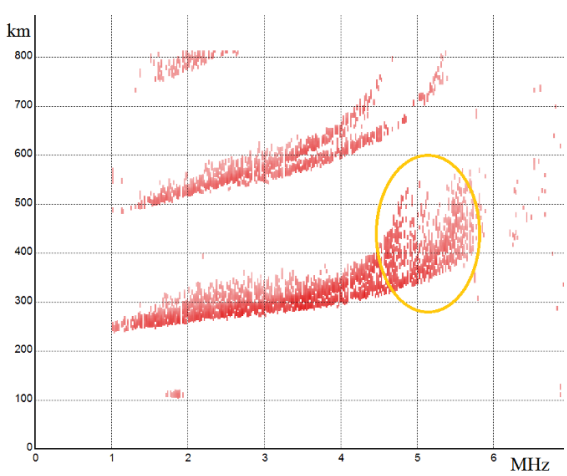

a)

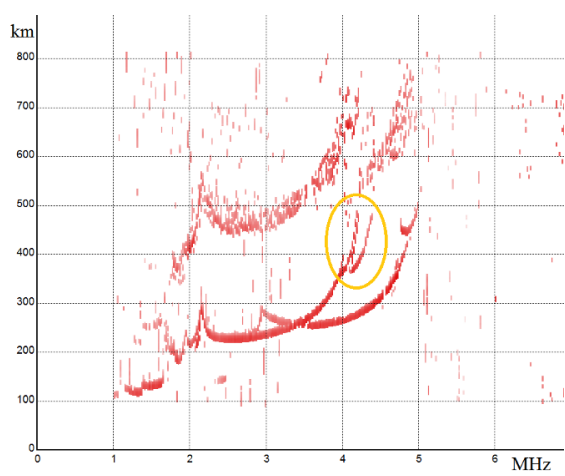

b)

Figure 1. Examples of anomalous behavior of ionospheric parameters formed on the background of a quiet magnetosphere: a) diffusion layer F2 (F2-spread) lasting several hours (mode "F"); b) stratification of layer F2 in frequency and height (mode "H", moving ionospheric disturbances)

Table 1 shows the time (UT) of ionospheric disturbances, and Fig. 2 shows the deviation of the critical frequency foF 2 from the median values and the change in the values of geomagnetic activity indices $K$ before the earthquake that occurred on 12.11 .2013 with the coordinates of the epicenter $\varphi=54.64^{\circ} \mathrm{N}, \lambda=162.44^{\circ} \mathrm{E}$, depth of hypocenter $h=67 \mathrm{~km}$ and magnitude $M=6.9$.

The short-term earthquake prediction algorithm presented in this paper is based on a joint analysis in a sliding time window with a width $\Delta T=5$ days with a step $\Delta t=1$ day 
Table 1. The duration of ionospheric disturbances during the period 09.11.2013-12.11.2013

\begin{tabular}{|c|c|c|c|c|}
\hline $\begin{array}{l}\text { ionospheric } \\
\text { disturbances }\end{array}$ & 09.11 .2013 & 10.11.2013 & 11.11 .2013 & 12.11 .2013 \\
\hline K-layer & $10: 15-11: 00$ & & $\begin{array}{l}09: 45-10: 00 \\
10: 30-10: 45\end{array}$ & \\
\hline Es-spread & & $06: 15-06: 30$ & & \\
\hline F-spread & & & 09:15-09:30 & 11:15-14:00 \\
\hline $\begin{array}{l}\text { The stratifica- } \\
\text { tion of the F2 } \\
\text { on frequency } \\
\text { and height }\end{array}$ & $22: 30-22: 45$ & & $\begin{array}{l}02: 15-02: 30 \\
08: 15-08: 30\end{array}$ & $\begin{array}{l}01: 15-01: 30 \\
03: 30-03.45 \\
04: 30-04: 45 \\
07: 15-07: 30 \\
08: 00-08: 15\end{array}$ \\
\hline Es type $r$ & $15: 00-18: 15$ & 18:00-18:15 & $16: 00-18: 00$ & \\
\hline
\end{tabular}

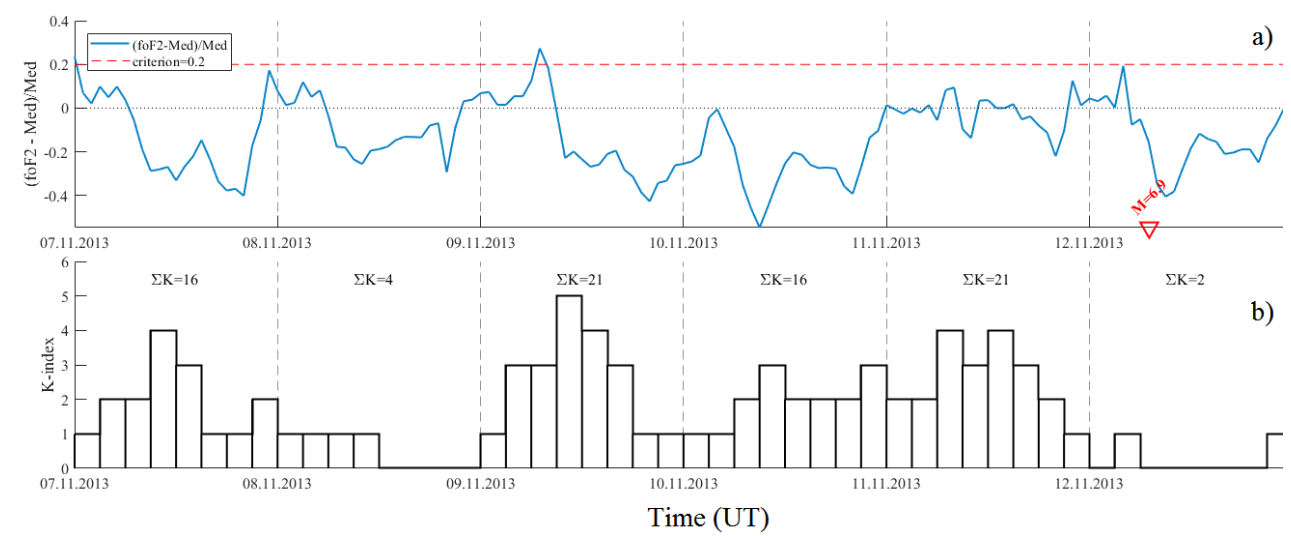

Figure 2. Time series for the period 07.11.2013-12.11.2013: a) relative deviations of the critical frequency $f \mathrm{oF} 2$ from its median values $f_{\text {med }}\left(\Delta f \mathrm{oF} 2 / f_{\text {med }}\right)$ b) three-hour values of the $K$-index.

of the following ionospheric parameters: the critical frequency $f \mathrm{oF} 2$ of the F2 layer, the manifestations of turbulence of the F2 and Es layers (F-spread and Es-spread), the F2 layer bundle in frequency and height (modes "H" and "V"), the formation of the corpuscular Klayer and the sporadic Es type $\mathrm{r}$ layer. The condition for declaring the beginning of the $t_{\mathrm{alarm}}$ alarm period was to perform the following criteria on the time interval $\Delta T$ for at least four considered ionospheric parameters:

- The excess of the critical frequency $f \mathrm{oF} 2$ of the F2 layer of the median fmed values calculated over the previous 30 days, not less than $20 \%\left(\Delta f \mathrm{oF} 2 / f_{\text {med }} \geq 0.2\right)$ on the background of the development of a magnetic storm (total $K$-index values for the day $\Sigma K \geq 20$ );

- Formation of the K-layer on the last day of the $\Delta T$ interval;

- The formation of the diffusion of sporadic Es layer (Es-spread);

- Formation of the diffusion layer F2 (F2-spread) during the last three days of the $\Delta T$ interval on the background of a quiet magnetosphere (three-hour values of the geomagnetic activity index during the day to $K \leq 2$ ); 
- Stratification of the F2 layer in frequency and height during the last three days of the $\Delta T$ interval against the background of a quiet magnetosphere $(K \leq 2)$;

- The formation of the sporadic Es layer of type $\mathrm{r}$ on the last day of the interval $\Delta T$.

The duration of the alarm period is set to $t_{\text {alarm }}=5$ days. The algorithm was tested on the time interval 01.01.2013-31.12.2017 in the spring and autumn seasons. As predicted seismic events were considered earthquakes of magnitude range $M \geq 5.0, M \geq 5.5, M \geq 6.0$, $M \geq 6.5$, which occurred at depths of $100 \mathrm{~km}$ in radius $r=500 \mathrm{~km}$ from the registration point of ionospheric observation IKIR FEB RAS in Kamchatka. The catalogue of earthquakes of the Kamchatka branch of the Federal Research Center "Unified Geophysical Service of RAS" [7] for 2013-2017 was used for the analysis.

\section{Efficiency evaluation of the forecast method}

Two approaches of A. A. Gusev [8] and G. M. Molchan [9] were used to assess the efficiency of the considered forecast method.

The reliability of the precursor $R$ is defined as the ratio of the number of earthquakes $n\left(E_{A}\right)$ for which the precursor was allocated to the number of all earthquakes $n(E)$ :

$$
R=\frac{n\left(E_{A}\right)}{n(E)}
$$

The validity of the precursor is defined as the ratio of the number of precursor anomalies $n\left(A_{E}\right)$ to the total number of isolated anomalies $n(A)$ :

$$
V=\frac{n\left(A_{E}\right)}{n(A)}
$$

According to the results presented in Table 2, the reliability of the precursor under consider-

Table 2. . Dependence of the reliability and validity of the precursor on the lower threshold of the considered earthquake magnitudes $M$.

\begin{tabular}{|c|c|c|c|c|}
\hline Magnitude $M$ & $M \geq 5.0$ & $M \geq 5.5$ & $M \geq 6.0$ & $M \geq 6.5$ \\
\hline$n\left(E_{A}\right)$ & 34 & 14 & 9 & 4 \\
\hline Missing earthquakes & 66 & 28 & 8 & 2 \\
\hline$n\left(A_{E}\right)$ & 34 & 14 & 9 & 4 \\
\hline False anomalies & 57 & 77 & 82 & 87 \\
\hline Reliability of the precursor $R$ & 0.34 & 0.33 & 0.53 & 0.67 \\
\hline Validity of the precursor $V$ & 0.37 & 0.15 & 0.1 & 0.04 \\
\hline
\end{tabular}

ation is higher for earthquakes of higher magnitudes than for smaller ones. For earthquakes with magnitude $M \geq 6.5$, the reliability of $R$ was 0.67 . The validity of the precursor $V$ takes the highest values for an earthquake with a magnitude $M \geq 5.0$ and is 0.34 . Since the precursor can be assigned to several earthquakes (the group) and should be considered when deciding about the implementation of forecasting and estimating the time of trouble, it was applied the following approach: 1) forecast is the realized first earthquake forecast magnitude appropriate interval, 2) the alarm is removed, 3) next earthquakes, which would formally correspond to the same precursor anomalies are declared missing ("skip goal") [10].

The efficiency of the forecast by the A. A. Gusev's method is calculated for a specific spatial region and a certain energy range of earthquakes by the formula:

$$
J_{G}=\frac{N_{+} / T_{\text {alarm }}}{N / T}
$$


where $T$ is the total time for monitoring the seismic situation; $N_{+}$is the number of earthquakes corresponding to the successful forecast for time $T ; N$ is the total number of earthquakes occurred (having spatiotemporal characteristics similar to those predicted) that occurred during time $T ; T_{\text {alarm }}$ is the total alarm time (the total duration of all periods of time in which the forecast was valid according to the estimated method during the total monitoring time). The statistical significance of $\alpha$ is determined by the probability of obtaining the $J_{G}$ efficiency values in the absence of the "earthquake-precursor"relationship. In the absence of such a connection, i.e. at random guessing, the efficiency of $J_{G}$ is 1 . As can be seen from the results

Table 3. Efficiency evaluation of the precursor by A. A. Gusev's method.

\begin{tabular}{|c|c|c|c|c|}
\hline Magnitude $M$ & $M \geq 5.0$ & $M \geq 5.5$ & $M \geq 6.0$ & $M \geq 6.5$ \\
\hline$N_{+}$ & 34 & 14 & 9 & 4 \\
\hline$N$ & 100 & 42 & 17 & 6 \\
\hline$T$ (day) & 1055 & 1055 & 1055 & 1055 \\
\hline$T_{\text {alarm }}($ day) & 228 & 272 & 282 & 286 \\
\hline Efficiency $J_{G}$ & 1.57 & 1.29 & 1.98 & 2.46 \\
\hline$\alpha$ & 0.003 & 0.28 & 0.023 & 0.049 \\
\hline
\end{tabular}

presented in Table 3, the $J_{G}$ efficiency takes the highest values for the range of predicted magnitudes $M \geq 6.0$ and $M \geq 6.5$. At the same time, the statistical significance of $\alpha$, which is 0.023 and 0.049 , respectively, demonstrates a low probability of accidental obtaining of such values in the absence of an earthquake connection with the considered precursor.

The efficiency of the precursor $J_{M}$ by G.M. Molchan's method is defined as

$$
J_{M}=1-v-\tau
$$

where $\tau=T_{\text {alarm }} / T$ is the alarm measure, $v=1-N_{+} / N$ is the proportion of target misses. For random prediction $J_{M}=0$, and for ideal (without missing target and with zero alarm time) $-J_{M}=1$. Table 4 presents the $J_{M}$ values for the four earthquake threshold magnitudes $M \geq 5.0, M \geq 5.5, M \geq 6.0, M \geq 6.5$. For a high threshold of magnitudes ( $M \geq 6.5$ ), the efficiency of $J_{M}$ is significantly (almost 3.3 times) higher than for a low threshold $(M \geq 5.0)$, which is mainly determined by the change in the reliability of the precursor.

Table 4. Efficiency evaluation of the precursor by G.M.Molchan's method.

\begin{tabular}{|c|c|c|c|c|}
\hline Magnitude $M$ & $M \geq 5.0$ & $M \geq 5.5$ & $M \geq 6.0$ & $M \geq 6.5$ \\
\hline$\tau$ & 0.22 & 0.26 & 0.27 & 0.27 \\
\hline$v$ & 0.66 & 0.67 & 0.47 & 0.33 \\
\hline Efficiency $J_{M}$ & 0.12 & 0.07 & 0.26 & 0.4 \\
\hline
\end{tabular}

Figure 3 shows Molchan's diagrams for the considered magnitude thresholds. In these diagrams the abscissa of the point is defined as a measure of anxiety $\tau$, and the ordinate is defined as the fraction of the passes of the target $v$. The diagonal of Molchan's diagram is $\tau+v=1$, connecting points $(0 ; 1)$ (the point of "optimist") and $(1 ; 0)$ (the point of "pessimist") corresponds to a random prediction. For this diagonal, we can construct a confidence interval with a given value $\alpha$. In this case, only the lower branch of this confidence interval is of interest. As follows from figure 3, the values $(\tau, v)$ obtained for the ranges of magnitudes $\geq 5.0, M \geq 6.0, M \geq 6.5$ lie under the lower limit of $99 \%$ of the confidence interval, which can be interpreted as a high degree of reliability of the revealed connection of the considered precursor with earthquakes of this range of magnitudes occurring at distances up to $500 \mathrm{~km}$ from the observation point. 


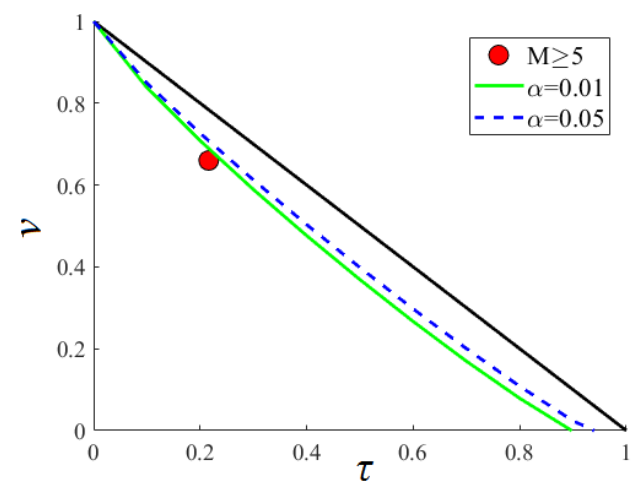

a)

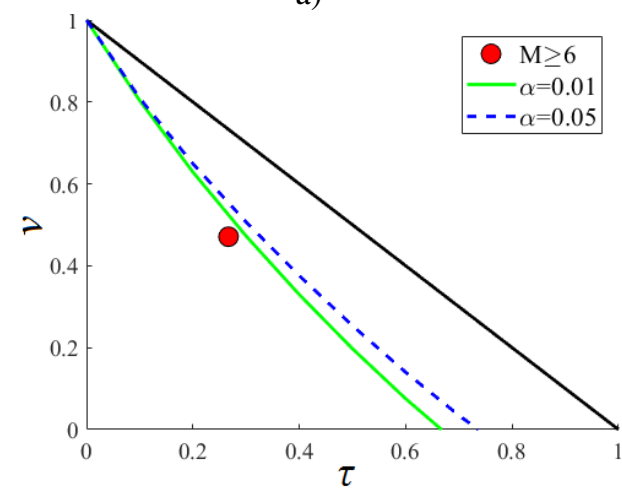

c)

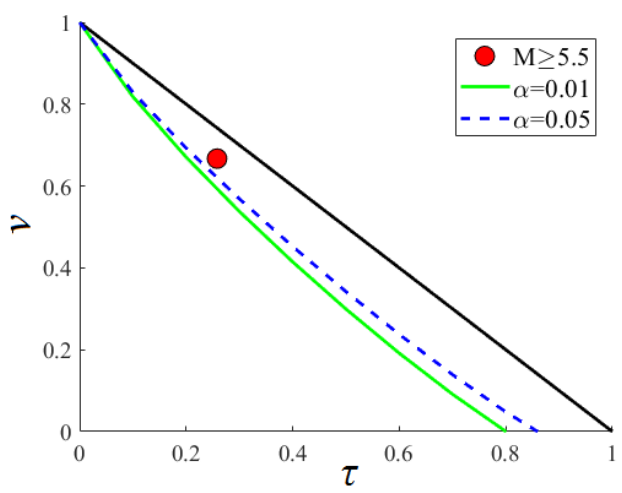

b)

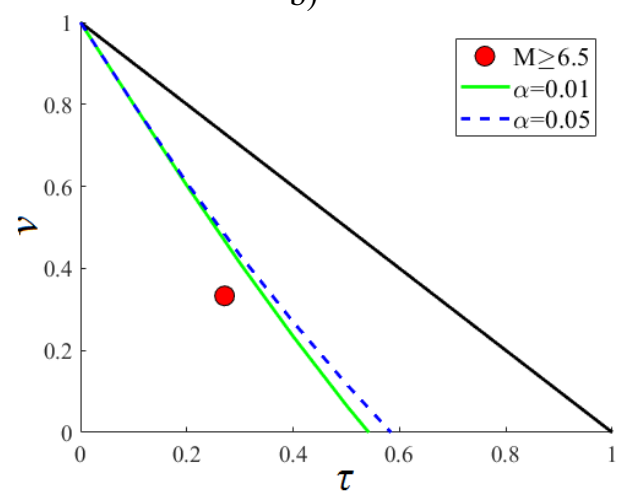

d)

Figure 3. Molchan's diagrams for different thresholds of magnitude $M$. The lower limits of the confidence interval of the random prediction with the significance level $\alpha=0.01$ and $\alpha=0.05$ are marked.

\section{Conclusion}

The method of short-term prediction of strong earthquakes is presented, in which the excess of the current values of the critical frequency $f \mathrm{oF} 2$ of the ionospheric layer $\mathrm{F} 2$ over the median values during the perturbed state of the magnetosphere, as well as the appearance of ionospheric disturbances: K-layer, Es-spread, F-spread, stratification layer F2, Es type r are considered as precursors. It is shown that the reliability of the forecast increases with the magnitude of the predicted earthquake. When predicting earthquakes with a magnitude $M \geq 6.0$, the reliability is 0.53 (i.e. $53 \%$ of earthquakes had a precursor), and the validity is 0.1 (i.e., $10 \%$ of the identified anomalies were realized). For earthquakes with $M \geq 6.5$ magnitude, the reliability was 0.67 and the validity was 0.04 . The effeciency of the precursor was evaluated in two ways. It is shown that the results of the application of the prognostic method are significantly different from the random guessing and give a gain of almost 2 times for $M \geq 6.0$ and almost 2.5 times for $M \geq 6.5$.

The work was carried out according the project FEB RAS \#18-5-095 "Development of new methods of complex geophysical monitoring in the purpose to predict strong earthquakes and prevent accidents in industrial electroenergy systems", section 1 of Complex Program of Fundamental scientific researches FEB RAS 2018-2020. 
The work was carried out by the means of the Common Use Center "North-Eastern Heliogeophysical Center "CKP_558279".

\section{References}

[1] V.M. Sorokin, V.M. Chmyrev, O.A. Pokhotelov, V.A. Liperovsky, Kratkij prognoz katastroficheskih zemletrjasenij s pomoshh'ju radiofizicheskih nazemno-kosmicheskih metodov (OIFZ RAS, Moscow, 1998) 64-85

[2] E.V. Liperovskaya, V.V. Bogdanov, P.-F. Biagi, C.-V. Meister, V.A. Liperovsky, M.V. Rodkin, Nat. Hazards Earth Syst. Sci. 9, 1-7 (2009)

[3] E.V. Liperovskaya, V.V. Bogdanov, P.-F. Biagi, C.-V. Meister, V.A.Liperovsky, Nat. Hazards Earth Syst. Sci. 8, 1807-1812 (2011)

[4] V.V. Bogdanov, A.V. Buzevich, A.V. Vinitsky, G.I. Druzhin, A.V. Kuptsov, I.N. Poddelsky, S.E. Smirnov, B.M. Shevtsov, Complex seismological and geophysical research of Kamchatka. To the 25th anniversary of the Kamchatka Experimental-Methodical Seismological Department of the GS RAS (KEMSD GS RAS, Petropavlovsk-Kamchatsky, 2004) 259-278

[5] V.V. Bogdanov, A.V. Kaisin, A.V. Pavlov, A.L. Polyukhova, C.-V. Meister, J. Physics and Chemistry of the Earth 98, 154-160 (2017)

[6] V.V. Bogdanov, A.V. Pavlov, E3S Web of Conferences 20, 03001 (2017)

[7] URL: http://www.emsd.ru/sdis/earthquake/catalogue/catalogue.php

[8] A.A. Gusev, Seismicity and seismic forecast, properties of the upper mantle and their relation to volcanism on Kamchatka (Nauka, Novosibirsk, 1974) 109-119

[9] G.M. Molchan, Physics of the Earth and Planetary Interiors 61, 84-98 (1990)

[10] V.A. Saltykov, Physics of the Earth 2, 84-96 (2017) 